\title{
A Knowledge Base System Project for FO(.)
}

\author{
Marc Denecker \\ Department Computer Science, Katholieke Universiteit Leuven \\ Celestijnenlaan 200A, B-3001 Heverlee, Belgium \\ marc.denecker@cs.kuleuven.be
}

\begin{abstract}
This talk reports on a project to build a Knowledge Base System (KBS) equipped with several forms of inference able to solve different sorts of tasks using the KB. The logic $\mathrm{FO}($.$) used in the KBS$ is an extension of classical logic (FO) with various language primitives such as inductive definitions, aggregates, arithmetic, etc. The logic is a natural integration (and further extension) of classical logic and logic programming, and is based on the view of a logic program as a definition. We discuss informal and formal semantics of definitions in $\mathrm{FO}($. and consider the relationship with other knowledge principles such as coinduction, the closed world assumption and causality and with the LP formalisms ASP, ALP and deductive databases. On the computational level, we will report on current attempts to build finite domain inference systems for model expansion, approximate reasoning, theory debugging and model revision, with special focus on the IDP-system, a model expansion system for $\mathrm{FO}($.$) .$
\end{abstract}

